\title{
Heparinization and hybridization of electrospun tubular graft for improved endothelialization and anticoagulation
}

\author{
Quanchao Zhang ${ }^{a}$, Shan $\mathrm{He}^{\mathrm{a}}$, Xiangbo Zhu ${ }^{\mathrm{a}}$, Honglin Luo ${ }^{\mathrm{a}, \mathrm{b}, *}$, Miguel Gama ${ }^{\mathrm{c}}$, Mengxia Peng ${ }^{\mathrm{a}}$, \\ Xiaoyan Deng ${ }^{a, * *}$, Yizao Wan ${ }^{\text {a,b,* }}$ \\ a Jiangxi Key Laboratory of Nanobiomaterials, Institute of Advanced Materials, East China Jiaotong University, Nanchang 330013, China \\ ${ }^{\mathrm{b}}$ School of Materials Science and Engineering, Tianjin University, Tianjin 300072, China \\ ${ }^{c}$ Centro de Engenharia Biológica, Universidade do Minho, Campus de Gualtar, P 4715-057 Braga, Portugal
}

\section{A R T I C L E I N F O}

\section{Keywords:}

Heparin

Bacterial nanocellulose

Vascular graft

Endothelialization

Anticoagulation

\begin{abstract}
A B S T R A C T
Constructing biomimetic structure and immobilizing antithrombus factors are two effective methods to ensure rapid endothelialization and long-term anticoagulation for small-diameter vascular grafts. However, few literatures are available regarding simultaneous implementation of these two strategies. Herein, a nano-micro-fibrous biomimetic graft with a heparin coating was prepared via a step-by-step in situ biosynthesis method to improve potential endothelialization and anticoagulation. The 4-mm-diameter tubular graft consists of electrospun cellulose acetate (CA) microfibers and entangled bacterial nanocellulose (BNC) nanofibers with heparin coating on dual fibers. The hybridized and heparinized graft possesses suitable pore structure that facilitates endothelia cells adhesion and proliferation but prevents infiltration of fibrous tissue and blood leakage. In addition, it shows higher mechanical properties than those of bare CA and hybridized CA/BNC grafts, which match well with native blood vessels. Moreover, this dually modified graft exhibits improved blood compatibility and endothelialization over the counterparts without hybridization or heparinization according to the testing results of platelet adhesion, cell morphology, and protein expression of von Willebrand Factor. This novel graft with dual modifications shows promising as a new small-diameter vascular graft. This study provides a guidance for promoting endothelialization and blood compatibility by dual modifications of biomimetic structure and immobilized bioactive molecules.
\end{abstract}

\section{Introduction}

Cardiovascular diseases (CVDs) cause millions of deaths per year which account for nearly $1 / 3$ of worldwide total deaths. Currently, conducting revascularization surgery to replace the diseased blood vessels with autologous or allogenic grafts is the most frequently recommended treatment. Although high patency rate can be realized through these treatments, supply shortage of these grafts motivates the development of alternative grafts. In this context, many artificial vascular grafts have been developed using polyethylene terephthalate (Dacron) [1] and expanded polytetrafluoroethylene (ePTFE) [2]. Indeed, these synthetic grafts are successfully applied as large diameter (larger than $6 \mathrm{~mm}$ ) blood vessel substitutes. However, unsatisfactory outcomes are noted when used as small-diameter (less than $6 \mathrm{~mm}$ ) vascular grafts due to thrombosis and intimal hyperplasia [3]. To this end, much work has been done to seek suitable materials for smalldiameter grafts and considerable improvements in endothelialization and anticoagulation have been demonstrated [4-9]. Despite these advancements, no grafts are satisfactory in clinics. Therefore, further improvements are required.

Ideally, a graft should have biomimetic structure mimicking that of native blood vessels $[10,11]$. It is accepted that electrospinning is the most widely used technique to produce biomimetic grafts [12-15]. However, bare electrospun grafts have disadvantages: Firstly, the pores are larger than those in the basement membrane in tunica intima of natural blood vessels which contain nano $(1-100 \mathrm{~nm})$ and submicron

\footnotetext{
* Corresponding authors at: Jiangxi Key Laboratory of Nanobiomaterials, Institute of Advanced Materials, East China Jiaotong University, Nanchang 330013, China; School of Materials Science and Engineering, Tianjin University, Tianjin 300072, China.

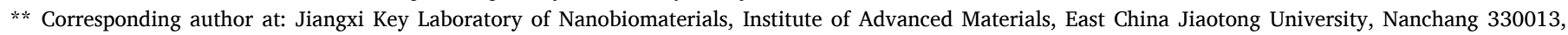
China.

E-mail addresses: hlluo@tju.edu.cn (H. Luo), dengxy1953@buaa.edu.cn (X. Deng), yzwan@tju.edu.cn (Y. Wan).
} 
(100-1000 nm) pores [16], leading to leakage of blood; Secondly, the fiber diameter in electrospun grafts is often several hundreds of nanometers, which cannot cover the fiber range from tens to hundreds nanometers of native blood vessels [15]. To this end, incorporating nano-scaled fibers into the electrospun grafts would be beneficial from the biomimetic viewpoint.

Apart from biomimetic structure, an ideal graft should also possess the ability to promote rapid endothelialization and prevent coagulation. An effective way is to conduct surface modification such that bioactive molecules and growth factors can be adsorbed and immobilized on the surface of fibrous grafts $[3,17,18]$. Among various bioactive molecules, heparin has gained considerable attention since it is an effective and widely used anticoagulant in clinics $[13,19,20]$. The use of heparin can not only minimize the formation of thrombus on artificial surfaces, but also inhibit the growth of smooth muscle cells [21]. However, there is no report in the literature regarding the dual promotions of incorporation of nano-scaled fibers into electrospun grafts and surface immobilization of heparin on dual fiber surfaces.

Herein, we report a novel electrospun microfibrous cellulose acetate (CA) tubular graft with incorporated bacterial nanocellulose (BNC) nanofibers, in which CA is an acetylated cellulose that can be electrospun into microfibers and BNC is a natural nanofibrous material with diameters ranging from $20 \mathrm{~nm}$ to $80 \mathrm{~nm}$, both being candidate materials for vascular grafts [22-25]. The CA microfibers and BNC nanofibers are surface immobilized with heparin and the resultant graft is abbreviated as CA/BNC-H. The incorporation of BNC into the CA electrospun graft was realized by step-by-step in situ biosynthesis [26-28] and heparin was immobilized onto dual fibers by grafting. The objectives of this work were to prepare the CA/BNC-H graft and determine how the incorporation of BNC nanofibers and immobilization of heparin affect the physiochemical properties of the CA electrospun graft and how blood platelets and endothelial cells (ECs) respond to the dual modifications.

\section{Materials and methods}

\subsection{Materials}

The CA with a weight-average molecular weight of $4.0 \times 10^{4} \mathrm{~g} / \mathrm{mol}$ was purchased from Shanghai Aladdin Bio-Chem Technology Co. Ltd., Shanghai, China. Absolute ethanol and acetic acid were provided by
Xilong Science Ltd., Shantou, China. The materials used for BNC biosynthesis (yeast extract, tryptone, disodium phosphate, and so on) were purchased from Beijing Innochem Science \& Technology Co., Ltd., China. All materials were used as received without further purification.

\subsection{Preparation of electrospun CA graft}

The electrospinning was conducted from a CA solution with a concentration of $15 \%(\mathrm{w} / \mathrm{v})$, which was prepared by dissolving CA into an acetone/acetic acid mixture ( $/ \mathrm{v}=1 / 1)$ under constant stirring. Electrospun CA graft was deposited on a cylinder receiver with a distance of $15 \mathrm{~cm}$ to the needle, an applied voltage of $10 \mathrm{kV}$, a rotating speed of 100 $\mathrm{rpm}$, a relative humidity of $40 \%$, and a mass flow rate of $5 \mathrm{~mL} / \mathrm{h}$ at ambient temperature. The length, inner diameter, and the wall thickness of the obtained CA graft was $30 \mathrm{~mm}, 4 \mathrm{~mm}$, and $1 \mathrm{~mm}$, respectively.

\subsection{Incorporation of BNC nanofibers into CA graft}

First, a BNC pellicle was prepared by conventional static culture method. The bacterial strains (Komagataeibacter xylinus X-2) and culture medium were reported previously [28,29]. The resultant BNC pellicle was used as substrate for the step-by-step in situ biosynthesis of BNC inside the porous CA graft [30]. The culture medium $(50 \mu \mathrm{L})$ was injected into the space between a silicone tube and the CA graft using a pipette gun to allow for in situ biosynthesis inside the CA graft (Fig. 1). The second injection of culture medium was followed $1 \mathrm{~h}$ after the in situ growth of $\mathrm{BC}$ within the CA scaffold at $37^{\circ} \mathrm{C}$. The injection and subsequent in situ biosynthesis was repeated 6 times at $1 \mathrm{~h}$ time intervals, yielding BNC nanofiber-incorporated CA (i.e., CA/BNC). The CA/BNC graft was obtained after boiling in $0.5 \mathrm{M} \mathrm{NaOH}$ solution, rinsing with deionized water to neutral, and freeze dried.

\subsection{Surface immobilization of heparin}

Typically, heparin powder was added to an 2-(4-Morpholino)ethanesulfonic acid (MES) solution with a heparin concentration of $0.5 \%$ (w/ v). Afterwards, N-(3-dimethylaminopropyl)-N'-ethylcarbodiimide (EDC) and N-hydroxysuccinimide (NHS) were added with a ratio of heparin:EDC:NHS $=1: 1: 0.6$. The CA/BNC graft was then immersed into the solution at $37{ }^{\circ} \mathrm{C}$ for $4 \mathrm{~h}$ under shaking. Subsequently, the heparin-

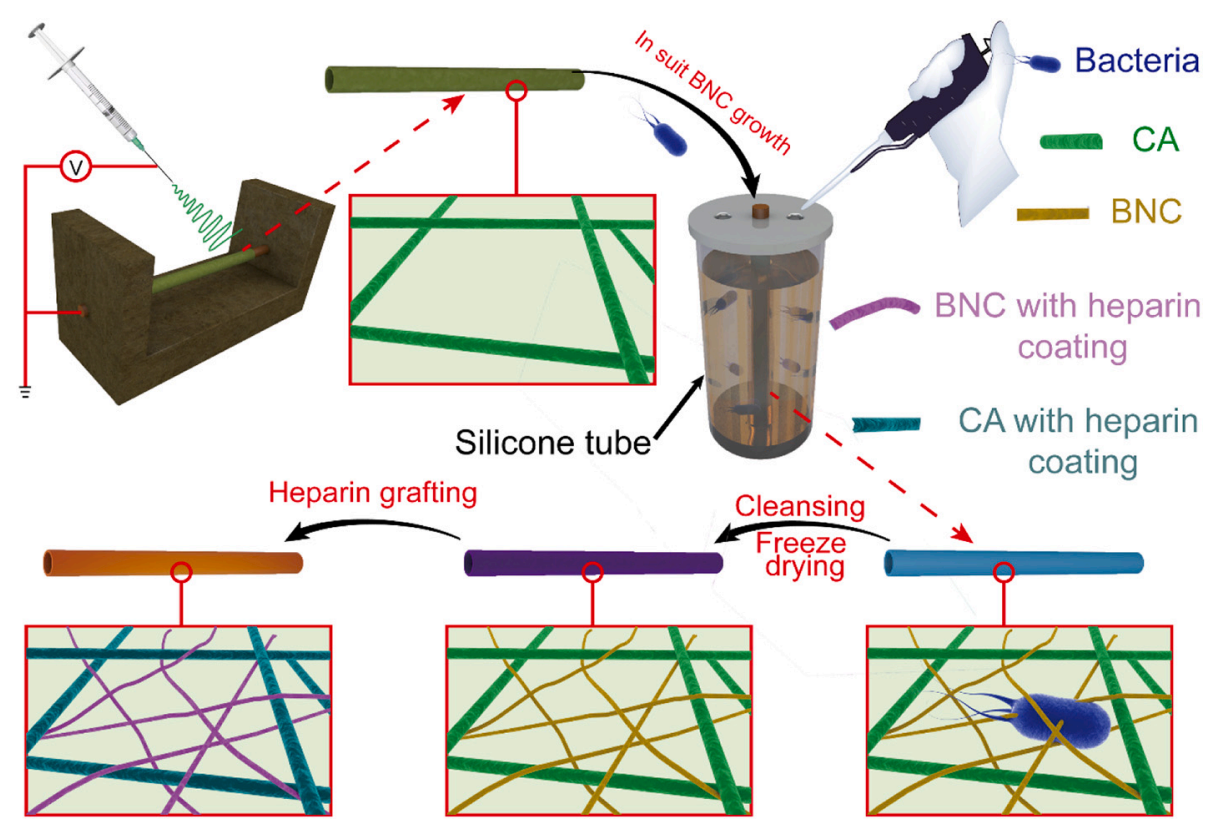

Fig. 1. Illustration of preparation of electrospun CA tubular graft with incorporated BNC nanofibers and surface heparinization. 
immobilized CA/BNC (namely CA/BNC-H) graft was washed with $0.1 \mathrm{M}$ $\mathrm{Na}_{2} \mathrm{HPO}_{4}$ twice to terminate the reaction. Then, $1 \mathrm{M} \mathrm{NaCl}$ solution was added to clean the graft 4 times and the solution was refreshed every $6 \mathrm{~h}$. Finally, the CA/BNC-H graft (Fig. S1, Supplementary Material) was obtained by rinsing with deionized water until neutral and freeze dried. The resultant product was named CA/BNC-H1. Other samples (CA/BNC$\mathrm{H} 2,3$, and 4) were produced using the same procedure with heparin concentrations of $1 \%, 3 \%$, and $5 \%(\mathrm{w} / \mathrm{v})$, respectively.

\subsection{Characterization}

A field emission scanning electron microscope (SEM, Hitachi, SU8010, Japan) was used to analyze the morphologies of CA, CA/BNC, and CA/BNC-H grafts. Fiber diameter and pore size were measured using Nano Measurer 1.2 software by randomly selecting at least 10 SEM images. Atomic force microscopy (AFM, NT-MDT Prima) was employed to observe the surface morphologies of grafts. An X-ray diffractometer (XRD, 6100, Shimazu, Japan) was used to assess the crystal structure under $40 \mathrm{kV}$ accelerating voltage and $30 \mathrm{~mA}$ current over a scanning range of $10-40^{\circ}$. To determine the surface chemistry, Fourier transform infrared spectrometer (FTIR, PerkinElmer, Spectrum one, USA) was used at a resolution of $4 \mathrm{~cm}^{-1}$. A thermal gravimetric analyzer (TGA, PerkinElmer, USA) was employed to test thermal stability under nitrogen flow. The samples were heated from 50 to $600{ }^{\circ} \mathrm{C}$ and the heating rate was set at $20{ }^{\circ} \mathrm{C} \mathrm{min}^{-1}$. A liquid/solid interface analyzer (Chengde Chenghui, JGM-360B) was used to measure the water contact angles.

Mechanical properties were tested using a precise miniature electromagnetic fatigue testing machine (MUF-1050, Tianjin Care Measure \& Control Co., Ltd., Tianjin, China) under a strain rate of $5 \mathrm{~mm} \mathrm{~min}{ }^{-1}$. The testing samples had the dimension of $10 \mathrm{~mm} \times 5 \mathrm{~mm} \times 2 \mathrm{~mm}$.

\subsection{Release of heparin}

To quantitatively determine the heparin release from CA/BNC-H grafts, the sterilized samples (1 $\mathrm{mm}$ in thickness and $15 \mathrm{~mm}$ in diameter) were immersed in $2 \mathrm{~mL}$ of PBS (phosphate buffered saline) solution and incubated at $37{ }^{\circ} \mathrm{C}$ under shaking at $120 \mathrm{rpm}$ for $1,2,3,5,7,10,16$, 21,28 , and 35 days. At each time point, the release media (PBS containing released heparin) was collected and renewed with fresh PBS. The collected liquid was used to test the heparin concentration using a heparin sodium detection kit (100 T, Ruichu Bio, Shanghai).

\subsection{Evaluation of blood compatibility}

The blood compatibility was evaluated by platelet adhesion test using platelet rich plasma (PRP) that was prepared using a procedure as previously reported [30] and circular CA, CA/BNC, and CA/BNC-H samples ( $1 \mathrm{~mm}$ in thickness and $15 \mathrm{~mm}$ in diameter). To quantitatively compare the blood compatibility, platelet adhesion was first tested by lactic acid dehydrogenase (LDH) release assay. Briefly, $100 \mu \mathrm{L}$ of fresh PRP was pipetted onto the surface of each graft sample and incubated at $37{ }^{\circ} \mathrm{C}$ for $2 \mathrm{~h}$. After removal of the PRP suspension, the samples were washed with PBS three times. Then, $150 \mu \mathrm{L}$ of the lysis solution was dropped on the sample surfaces and incubated at $37{ }^{\circ} \mathrm{C}$ for $1 \mathrm{~h}$. Subsequently, a mixture of $120 \mu \mathrm{L}$ of lysate and $80 \mu \mathrm{L}$ of substrate solution was obtained and then centrifuged at $4000 \mathrm{rpm}$ for $5 \mathrm{~min}$. The supernatant was collected and the absorbance was read at $490 \mathrm{~nm}$ using a microplate reader (iMark, Bio Rad, California, USA). At the same time, the platelets adhered on sample surfaces were observed using the aforementioned SEM after fixation with $2.5 \mathrm{wt} \%$ glutaraldehyde solution, dehydration with a series of ethanol solution, air drying, and gold sputtering.

\subsection{Assessment of cytocompatibility}

Circular samples of CA, CA/BNC, and CA/BNC-H were sterilized at
$121{ }^{\circ} \mathrm{C}$ for $30 \mathrm{~min}$ and placed in a 24 -well plate. The human umbilical vein endothelial (HUVEC) cells were seeded on the graft samples at a density of $1 \times 10^{4}$ cells per well. The incubation was carried out in endothelial cell medium (ENCM) supplemented with $10 \%$ fetal bovine serum (FBS) and $1 \%$ endothelial cell growth supplement (ECGS) for 1, 4, and 7 days at $37{ }^{\circ} \mathrm{C}$ in a $5 \% \mathrm{CO}_{2}$ environment. Upon completion of incubation, a routine Cell Counting Kit-8 (CCK-8) assay was conducted to assess the proliferation by measuring the absorbance at $450 \mathrm{~nm}$ using the aforementioned microplate reader, as reported previously [30].

Fluorescent cell images were obtained using live/dead assay. After incubation for 4 and 7 days under the aforementioned conditions and washing twice with PBS, cells on the samples were stained using a live/ dead staining reagent (fluorescein diacetate/PI, Solarbio, Beijing, China) in accordance with the manufacturer's instructions and then observed using a fluorescence inverted microscope (TS2, Nikon, Tokyo, Japan). For quantitation, representative images were collected, and cell number was analyzed using ImageJ software (National Institutes of Health, USA).

Cell morphologies on sample surfaces were examined using SEM after routine incubation, washing, fixation with $2.5 \mathrm{wt} \%$ glutaraldehyde, dehydration, freeze drying, and gold sputtering, as reported in our previous studies [30,31].

\subsection{Cytoskeleton, nuclei, and immunofluorescent staining}

To observe cytoskeleton and nuclei, cellular actin cytoskeleton was stained with $100 \mathrm{nM}$ of rhodamine phalloidin (Solarbio, Beijing, China) and cell nuclei were counter-stained with $5 \mu \mathrm{L} / \mathrm{mL}$ of 4,6-diamidino-2phenylindole (DAPI, Solarbio, Beijing, China) for $20 \mathrm{~s}$. The stained samples were then observed using a confocal laser scanning microscopy (CLSM, Leica SP8). For staining with the von Willebrand Factor (vWF), samples after 3 days of culture in 24-well plates filled with ENCM were fixed using $4 \%$ paraformaldehyde for $15 \mathrm{~min}$, permeabilized using $0.2 \%$ TritonX-100 in PBS for 15 min, and blocked using 2\% bovine serum albumin (BSA) for $40 \mathrm{~min}$ at $37{ }^{\circ} \mathrm{C}$ to reduce nonspecific staining. The blocked samples were stained using vWF (2\% BSA diluted primary antibody) and then Alexa 488 (second antibody, diluted at 1:100 in 2\% BSA). Finally, samples were washed, stained with rhodamine phalloidin for $30 \mathrm{~min}$ and DAPI for $30 \mathrm{~s}$, and observed using the same CLSM.

\subsection{Statistical analysis}

Statistical analysis was performed using a Statistical Product and Service Solutions (SPSS) software (version 20). A statistical significance was considered when $p$-value is less than 0.05 .

\section{Results}

\subsection{Characterization of $C A / B N C-H$ grafts}

SEM images (Fig. 2A) reveal the fibrous structure of CA, BNC, CA/ $\mathrm{BNC}$, and CA/BNC-H grafts. CA has thick electrospun fibers and BNC shows nanofibrous and porous morphology, in consistent with previous reports [31,32]. CA/BNC and CA/BNC-H show entangled dual fibers of thick CA fibers and fine BNC fibers. There is no significant difference in morphology between CA/BNC-H1, 2, 3, and 4 although their heparin contents are different (Fig. S2, Supplementary Material). SEM cannot tell the difference in morphology between CA/BNC and CA/BNC-H. Nevertheless, AFM images (Fig. 2B) clearly demonstrate rougher surface of CA/BNC-H3, as a model CA/BNC-H sample, as compared to CA and $\mathrm{CA} / \mathrm{BNC}$, which is due to the immobilization of heparin. The formation of a heparin layer on the surface of the graft was also observed by other researchers [33], which is important for the blood compatibility [34]. Fig. 2C and D reveals that incorporation of BNC decreases the average pore size of CA (CA $v s$. CA/BNC), while heparin immobilization does not cause significant changes in the average fiber and pore sizes 
(A)
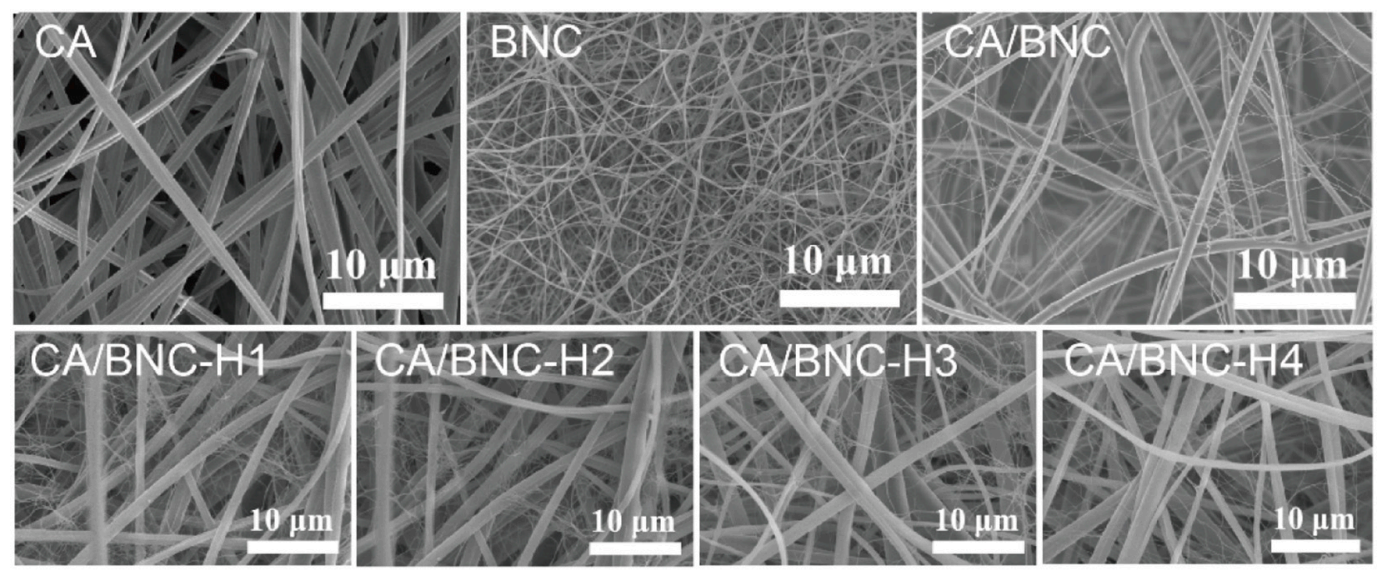

(B)

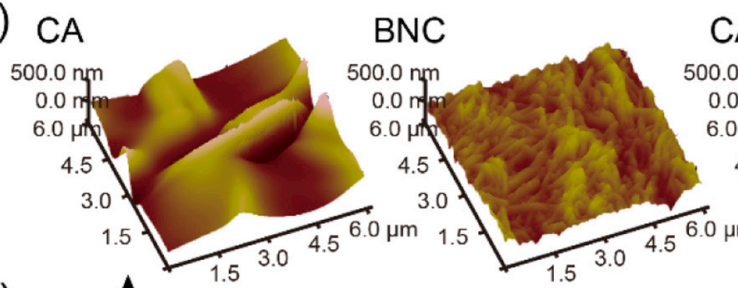

\section{CA/BNC}

(C)
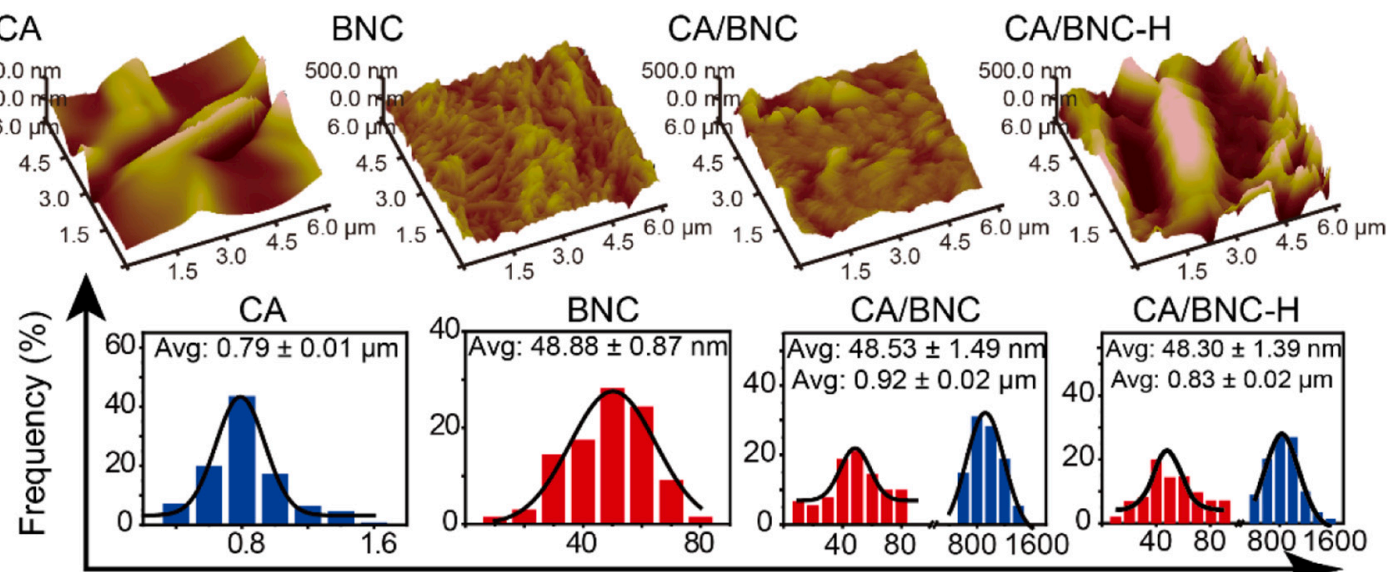

Fiber diameter $(\mathrm{nm})$

(D)

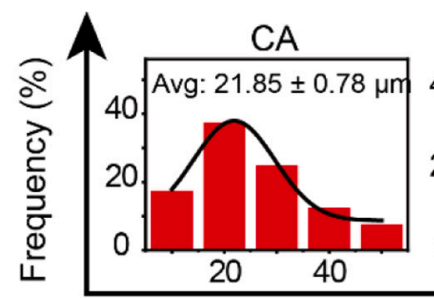

BNC

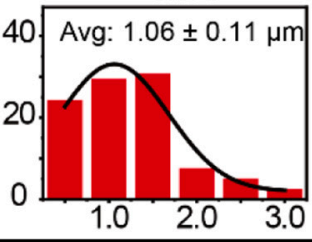

$\mathrm{CA} / \mathrm{BNC}$
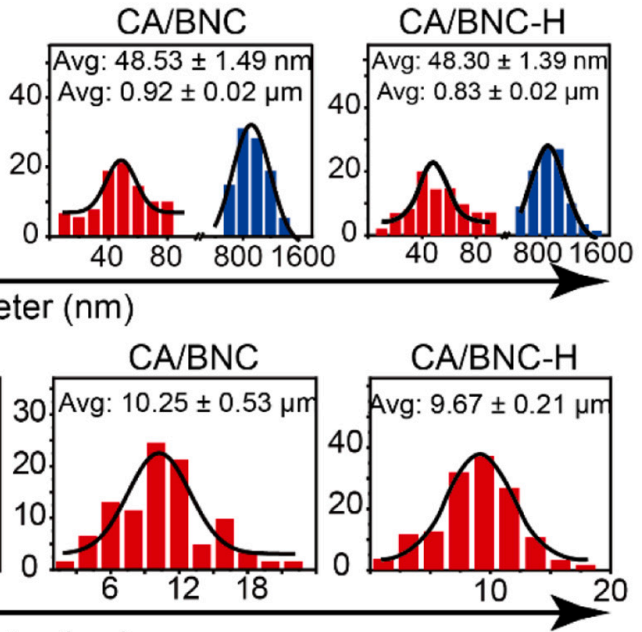

Pore diameter $(\mu \mathrm{m})$

Fig. 2. Morphologies of CA, BNC, CA/BNC, and CA/BNC-H grafts. (A) SEM micrographs. (B) AFM images. (C) Fiber diameter distribution. (D) Pore diameter distribution.

\section{(CA/BNC vs. CA/BNC-H).}

To determine the chemical state of heparin on BNC and CA fibers, $\mathrm{XRD}$ and FTIR analyses were conducted. As seen in Fig. 3A, three peaks at $14.2^{\circ}, 16.6^{\circ}$, and $22.6^{\circ}$ are observed, which are characteristic peaks of cellulose I [35]. There is a broad peak centered at around $22.6^{\circ}$ in the XRD pattern of CA, which is consistent with previous reports [36,37]. The XRD pattern of heparin is similar to that reported by Zhao et al. [38]. The XRD patterns of CA/BNC and CA/BNC-H are similar to that of bare BNC except for an additional peak at around $20^{\circ}$, which was also observed in our previous work [31].

Fig. 3B shows the FTIR spectra of CA, BNC, heparin, CA/BNC, and CA/BNC-H samples. All samples except heparin show a strong absorption peak at $2919 \mathrm{~cm}^{-1}$, which is attributed to the stretching vibration of $-\mathrm{CH}_{2}$. The absorption peaks at $1640 \mathrm{~cm}^{-1}$ and $1061 \mathrm{~cm}^{-1}$ are attributed to the stretching of $\mathrm{H}-\mathrm{O}-\mathrm{H}$ and the asymmetric stretching vibration of $\mathrm{C}$ O-C, respectively [39-41]. Note that CA presents a peak at $1740 \mathrm{~cm}^{-1}$, which is attributable to the stretching vibration of the ester group $\mathrm{C}=\mathrm{O}$ of CA molecule [42]. This peak is absent in the spectrum of CA/BNC due to the complete deacetylation by the $\mathrm{NaOH}$ treatment [31]. It is worth noting that the $-\mathrm{OH}$ peak of CA/BNC shifts from $3419 \mathrm{~cm}^{-1}$ in BNC to
$3498 \mathrm{~cm}^{-1}$, likely due to the hydrogen bond between CA and BNC [22], which is schematically illustrated in Fig. 3C. In the heparin spectrum, the absorption peaks at $807 \mathrm{~cm}^{-1}$ (axial deformation of C-O-S), 1061 $\mathrm{cm}^{-1}$ (asymmetric stretching vibration of C-O-C), $1239 \mathrm{~cm}^{-1}(\mathrm{~S}=\mathrm{O}$ axial deformation), $1540 \mathrm{~cm}^{-1}(\mathrm{~N}-\mathrm{H}$ bending vibration and $\mathrm{C}-\mathrm{N}$ tensile vibration) and $1640 \mathrm{~cm}^{-1}$ ( $\mathrm{C}=\mathrm{O}$ stretching vibration) as well as the broad band between 3600 and $3100 \mathrm{~cm}^{-1}$ (vibration stretching of $-\mathrm{OH}$ group) can be observed [43-45]. All these peaks can be found in the FTIR spectra of CA/BNC-H grafts, suggesting the presence of heparin in $\mathrm{CA} / \mathrm{BNC}-\mathrm{H}$ samples. Moreover, compared to $\mathrm{CA} / \mathrm{BNC}$, the peak intensity at $1640 \mathrm{~cm}^{-1}$ in CA/BNC-H grafts is stronger and it increases with heparin content, which is mainly ascribed to the formation of a new ester bond between heparin and CA/BNC [46] (Fig. 3C), indicating the successful grafting of heparin. Note that the $-\mathrm{OH}$ peak shows a gradual shift from $3441 \mathrm{~cm}^{-1}$ in CA/BNC to $3461 \mathrm{~cm}^{-1}$ in CA/BNC-H4, likely caused by the formation of hydrogen bond between heparin and CA/ BNC [47], as illustrated in Fig. 3C.

The TGA results (Fig. S3, Supplementary Material) of CA, BNC, heparin, $\mathrm{CA} / \mathrm{BNC}$, and $\mathrm{CA} / \mathrm{BNC}-\mathrm{H}$ demonstrate that all samples except heparin have three-stage curves. There are slight mass losses below 


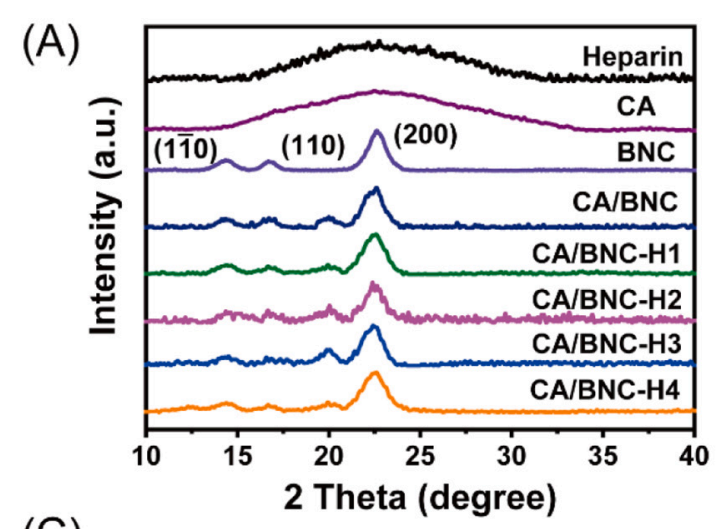

(C)

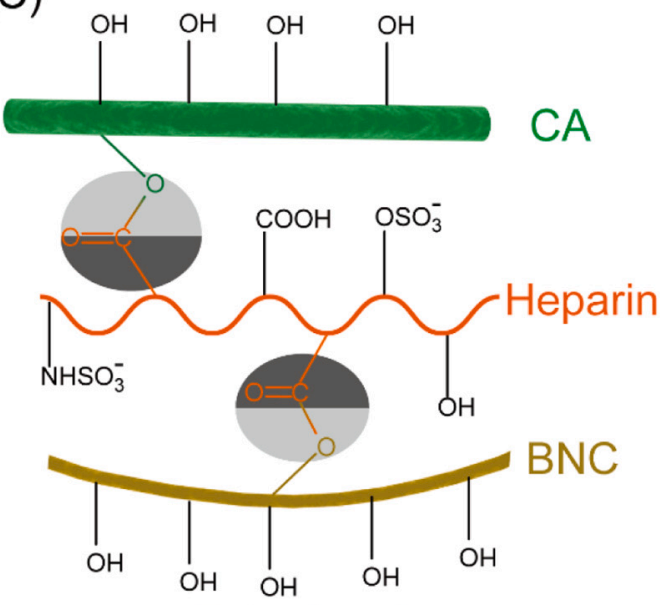

Ester bond
(B)
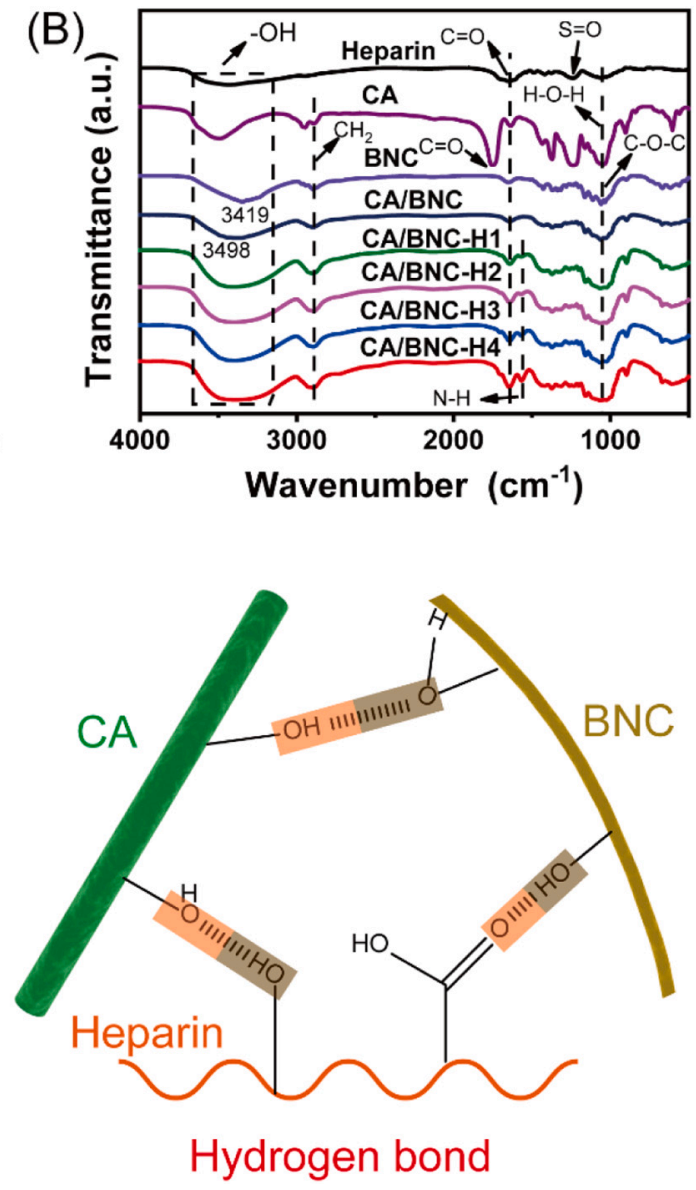

Fig. 3. XRD (A) and FTIR (B) spectra of BNC, CA, CA/BNC, heparin, and CA/BNC-H grafts. (C) Proposed interactions between BNC, CA, and heparin.

$275{ }^{\circ} \mathrm{C}$, mainly due to evaporation of physically adsorbed water [48]. Rapid mass losses are noted beyond $390{ }^{\circ} \mathrm{C}$, due to a series of decomposition processes such as chain depolymerization, dehydration, and pyrolysis of glucose [49]. It is worth noting that the water contents are different, among which heparin has the highest while BNC the lowest. The main reason is that heparin contains abundant hydrophilic groups such as carboxylate (-COO-) and sulfonate $\left(-\mathrm{OSO}_{3}-\right.$ ), which can strongly interact with water molecules through dipole-dipole or ion-dipole [50]. TGA results suggest the decrease of thermal stability of CA/BNC-H as compared to CA/BNC, due to the addition of thermally unstable heparin, and the reduction increases with heparin content.

Fig. S4 (Supplementary material) presents water contact angles of CA, BNC, CA/BNC, and CA/BNC-H samples. As expected, CA shows a very high contact angle of $121.2^{\circ}$ since it is hydrophobic. After incorporation of $\mathrm{BNC}$, a much lower contact angle $\left(77.3^{\circ}\right)$ is found in CA/BNC due to the presence of hydrophilic $\mathrm{BNC}$ and $\mathrm{NaOH}$ treatment as mentioned above. More importantly, CA/BNC-H samples show significantly lower contact angles (31.8-35.5 ${ }^{\circ}$ ) than that of CA/BNC due to the large number of hydrophilic groups on heparin, as displayed in FTIR spectra (Fig. 3B).

Fig. 4 presents the mechanical properties of BNC, CA, CA/BNC, and $\mathrm{CA} / \mathrm{BNC}-\mathrm{H}$ grafts. The incorporation of $\mathrm{BNC}$ to CA greatly increases the tensile strength (Fig. 4A) and Young's modulus (Fig. 4B) while the elongation at break (Fig. 4C) of CA/BNC is comparable to CA. More importantly, CA/BNC-H1, 2, 3, 4 samples show significantly higher tensile strength and elongation at break than CA/BNC $(p<0.05$ in each
(A)

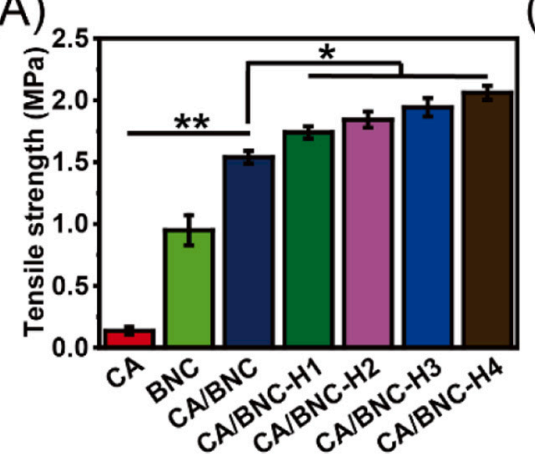

(B)

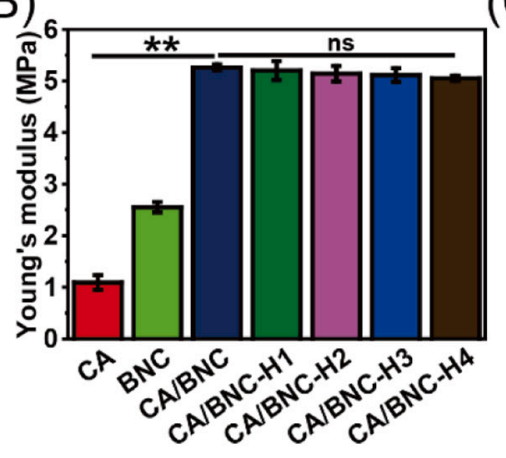

(C)

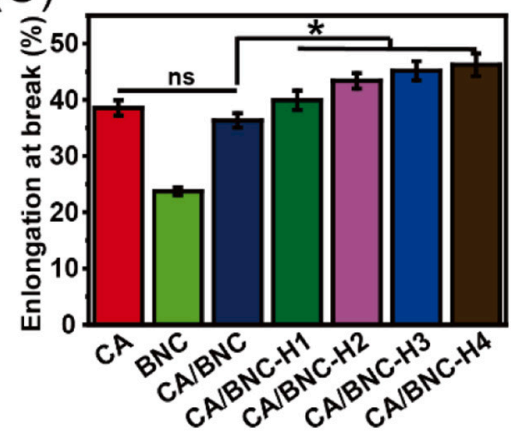

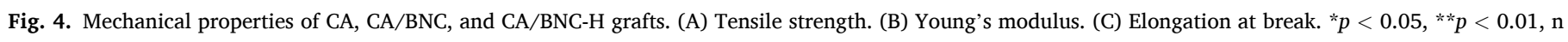
$=5$. 
case). This phenomenon was also reported by Lu et al. who claimed a significant increase (from $450 \pm 30 \mathrm{kPa}$ to $590 \pm 7 \mathrm{kPa}$ ) in compressive strength when heparin was added to a fibroin/collagen scaffold [51].

\subsection{Release of heparin from $C A / B N C-H$ grafts}

The in vitro release profiles of heparin are shown in Fig. 5. Similar to many heparin immobilized scaffolds $[13,51]$, there is an initial burst release of heparin from CA/BNC-H grafts. The release amounts from CA/ BNC-H3 are $18 \%, 37 \%$, and $55 \%$ on the 1 st, 3rd, and 10 th days, respectively. After 21 days, the release amount stabilizes and the heparin remained in CA/BNC-H3 is approximately $40 \%$. The comparisons between CA/BNC-H1, 2, 3, and 4 reveal that the remained amount is inversely proportional to the heparin content (Fig. S2) in the grafts.

\subsection{Evaluation of platelet adhesion}

To investigate the effects of heparinization and hybridization on antithrombogenic properties, platelet adhesion test was carried out to assess the platelet adhesion and activation on CA, CA/BNC, and CA/ BNC-H3 (as a representative CA/BNC-H sample) grafts. A few platelets are observed on CA (Fig. 6A) because it is hydrophobic (Fig. S4). When BNC is incorporated, the CA/BNC becomes hydrophilic and it is colonized by abundant platelets (Fig. 6B). Importantly, the platelets adhered on $\mathrm{CA} / \mathrm{BNC}$ show irregular shape, suggesting activation, which differs from those on CA/BNC-H3. A comparison between Fig. 6B and C clearly suggests that immobilization of heparin on the surface of BNC and CA fibers substantially inhibits platelet adhesion onto the surface of CA/ BNC-H3. The number of adhered platelets on CA/BNC is higher than that on CA, and approximately 2.5-fold higher than that on CA/BNC-H3 (Fig. 6D). Moreover, fewer platelets are adhered on CA/BNC-H3 as compared to CA. This result may suggest that CA/BNC-H3 has favorable antithrombogenic properties.

\subsection{Assessment of cytocompatibility and endothelialization}

To determine the effect of hybridization and heparinization on endothelialization, HUVEC cells were cultured on CA, CA/BNC, and CA/ BNC-H grafts. The cell adhesion and viability of HUVEC cells cultured on these grafts were evaluated using the live/dead staining and imaged with the fluorescence microscopy after incubation for 1, 4, and 7 days (Fig. 7A). Extensive cell population is observed across the surface of each graft and all grafts promote adhesion and growth of HUVEC cells after seeding for 4 days. Fig. 7B indicates that the density of living cells on $\mathrm{CA} / \mathrm{BNC}-\mathrm{H} 3$ is significantly higher as compared with CA and CA/BNC $(p$

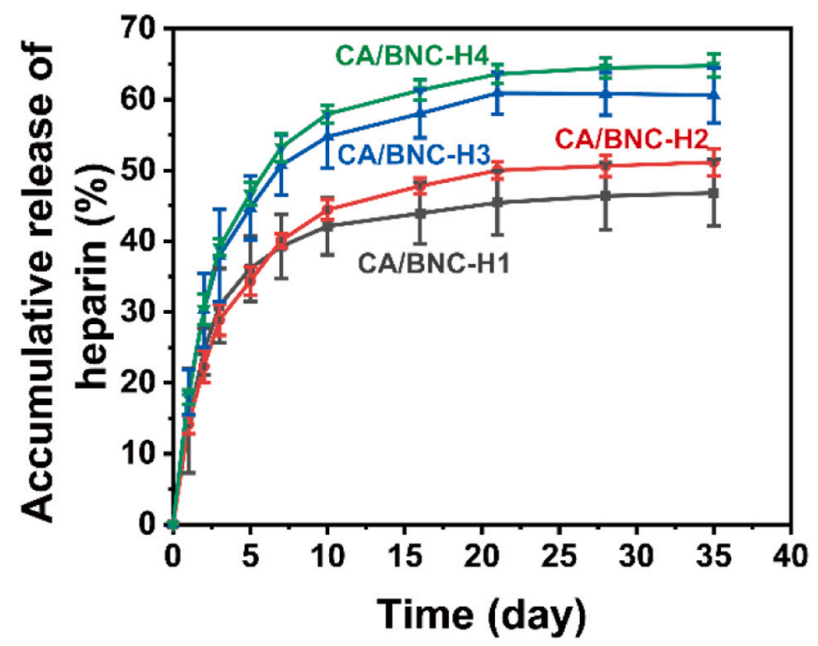

Fig. 5. Release profiles of heparin from CA/BNC-H grafts.
$<0.001)$.

SEM was used to observe the cell morphology on each graft. The cells on CA (Fig. 8A) are smoother than those on CA/BNC (Fig. 8B), while the cells on CA/BNC-H3 show abundant filopodia (Fig. 8C), suggesting that the cells grow better on CA/BNC-H3.

Quantitative cell viability and proliferation results are displayed in Fig. 8D. Although rapid cell proliferation is observed on each graft, cell viability (indicated by absorbance) is significantly different between CA, $\mathrm{CA} / \mathrm{BNC}$, and CA/BNC-H even on the first day. CA exhibits the lowest viability at each time point and a significant improvement is observed after hybridization with BNC fibers. Moreover, further increase in cell viability is observed when BNC and CA fibers are heparinized. These results reveal that both heparinization and hybridization enhance endothelialization of the CA graft.

To further assess endothelialization and coverage by HUVEC cells, Factin was stained with rhodamine phalloidin (red) and the nuclei were counter-stained with DAPI (blue), In addition, HUVEC cells were also stained for von vWF with green color. Fig. 9A shows the stained cells 3 days post seeding. Overall, the HUVEC cells proliferate well and a layer of endothelium is formed on each graft. In addition, HUVEC cells were also stained for von vWF with green color (Fig. 9B). The expression level of vWF on CA/BNC-H3 is higher than that on CA and CA/BNC.

\section{Discussion}

Developing an applicable small-diameter vascular graft remains a huge challenge due to vexed thrombosis and intimal hyperplasia [3]. Anticoagulation and rapid endothelialization are two requirements in designing small-diameter grafts [52]. It has been well accepted that complete endothelialization can prevent the formation of thrombosis and intimal hyperplasia and thus rapid endothelialization is critical to the success of vascular grafts $[53,54]$. To this end, numerous strategies have been developed to promote the attachment, proliferation, and phenotypic maintenance of ECs on the surface of grafts because these processes occur on the surface of native extracellular matrix (ECM). The first method is to fabricate grafts with a structure analogous to native blood vessels [30] that are composed of three layers of tunica intima, tunica media, and tunica adventitia [10,55]. More specifically, the artificial grafts should have a structure bearing resemblance to tunica intima consisting of nano- to micro-scaled fibers, which is covered with a monolayer of ECs and determines the anti-thrombosis [12,56]. The second method is surface modification with bioactive molecules such as heparin because, in natural blood vessels, factors released by ECs play a critical role in promoting antithrombogenic nature [57]. Although the above-mentioned methods have been adopted individually by many researchers, few studies have investigated the control of blood compatibility and endothelialization by integrating dual modifications of structure imitation and surface immobilization. The present study aims at determining the combined effects of hybridization and heparinization on blood compatibility and endothelialization.

To mimic the structure of native blood vessels, BNC nanofibers were hybridized with CA microfibers, leading to a graft with biomimetic structure. On the one hand, the hybridization results in greatly decreased pore size: The CA shows an average pore size of around $22 \mu \mathrm{m}$ with the largest pore size of $50 \mu \mathrm{m}$ (Fig. 2). In sharp contrast, CA/BNC and CA/BNC-H3 presents a porous structure with an average pore diameter of around $10 \mu \mathrm{m}$ (Fig. 2), which is smaller than the size of fibroblasts (approximately $15 \mu \mathrm{m}$ [58]). Such pore size is believed to allow EC adhesion and proliferation and communication between ECs and smooth muscle cells (SMCs), while preventing fibrous tissue infiltration and blood leakage [58,59]. In other words, CA/BNC-H3 graft has a suitable pore size that facilitates endothelialization while minimizing the risk of fibrous tissue infiltration and blood leakage [54]. On the other hand, the hybridization also endows the CA/BNC and CA/BNC-H3 with biomimetic nano-micro-sized fibers. Our previous studies suggested that the graft with nano-micro-sized fibers presented enhanced 

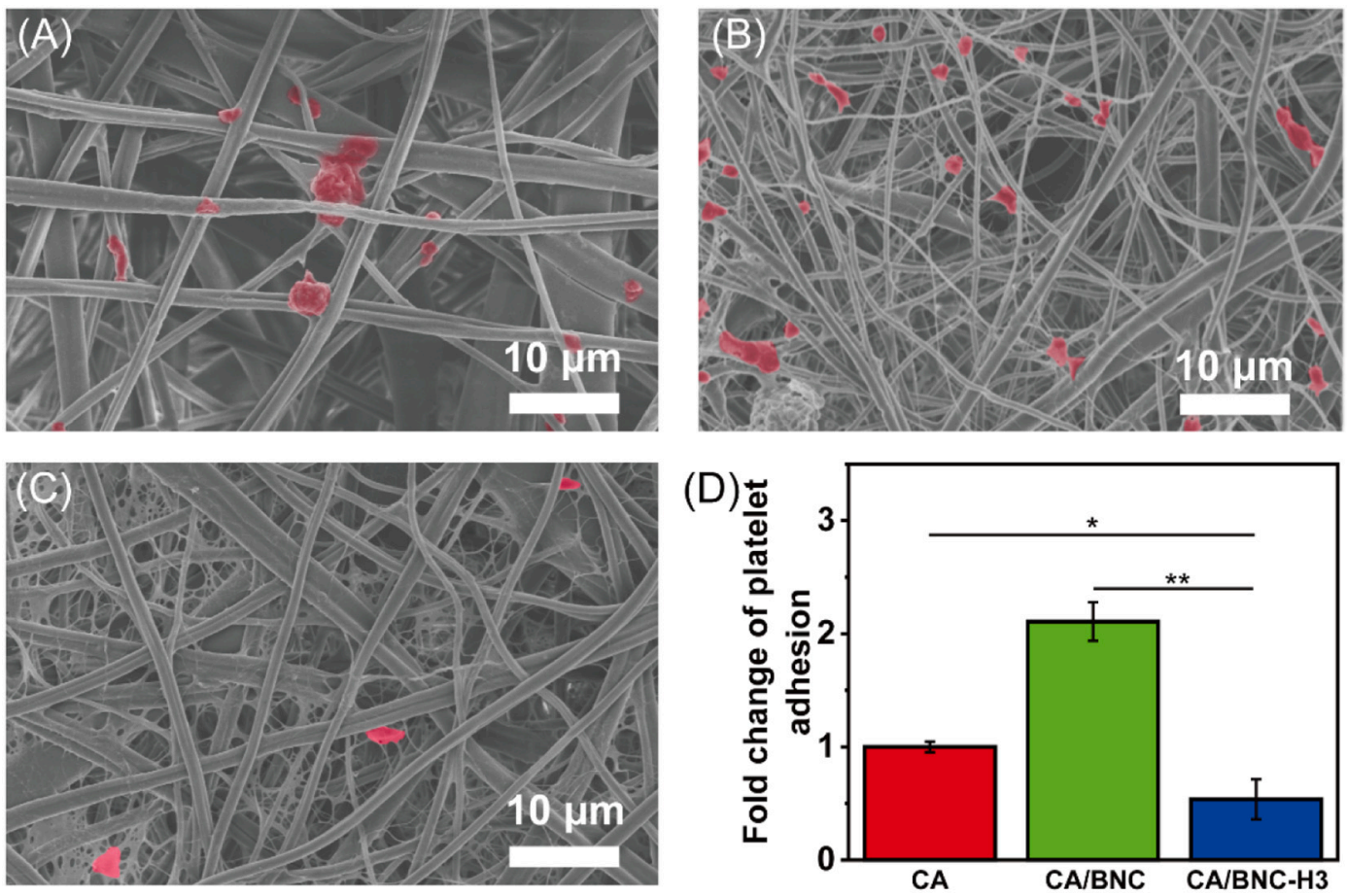

Fig. 6. (A-C) SEM images of platelets adhered on CA (A), CA/BNC (B), and CA/BNC-H3 (C) grafts. (D) Quantification of adhered platelets measured by lactate dehydrogenase (LDH) activity after incubation of CA, CA/BNC and CA/BNC-H grafts in platelet-rich plasma (PRP). ${ }^{*} p<0.05,{ }^{* *} p<0.01, \mathrm{n}=5$.
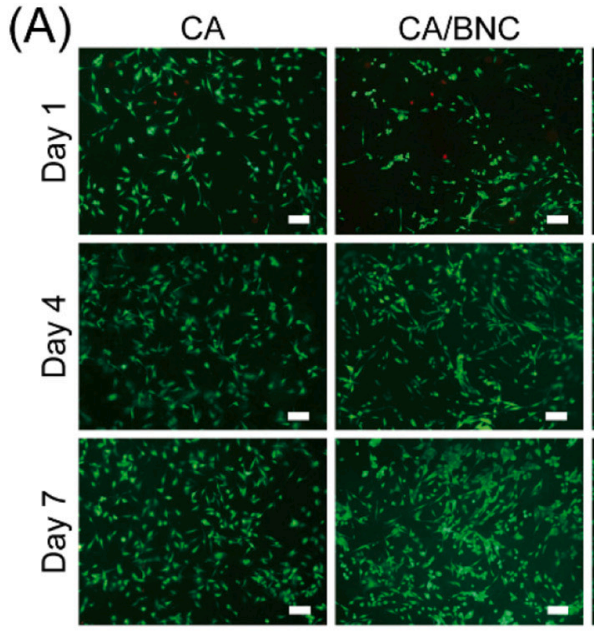

CA/BNC-H3

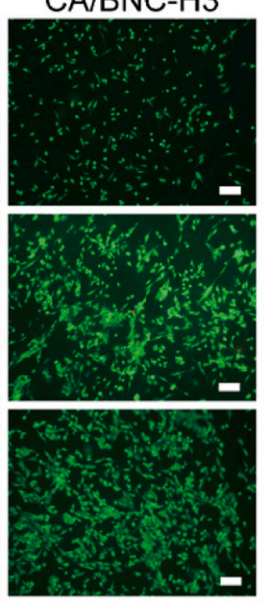

(B)

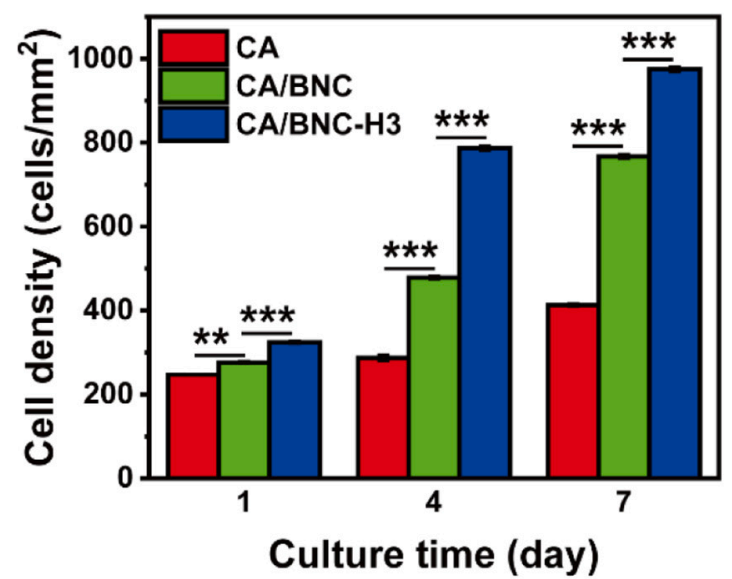

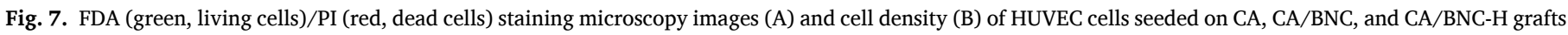

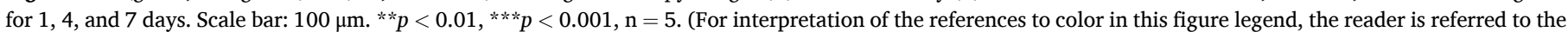
web version of this article.)

endothelialization over the microfiber-only CA graft [30], which is consistent with the results presented in Fig. 7 that demonstrates more cells on CA/BNC than CA. This further suggests that hybridization can promote cell attachment and proliferation by providing biomimetic microenvironment for cells. Importantly, Fig. 7 also confirms that the largest cell number is observed on CA/BNC-H3, suggesting that heparin immobilization on a graft consisting of nano-micro-fibers can immensely promote EC attachment and proliferation, agreeing with previous studies [60,61]. According to our results, hybridization and heparinization can effectively enhance the adhesion and viability of ECs and the combined effect is much higher than sole hybridization. In other words, there are synergistic effects between hybridization and heparinization in favoring endothelialization.
We also evaluate cell attachment and proliferation by observing the cytoskeleton and nuclei organization of HUVEC cells (Fig. 9A). The HUVEC cells seeded on CA/BNC-H3 exhibit a wider spreading as compared to CA and CA/BNC. These results further suggest that hybridization and heparinization promote endothelialization. Furthermore, we measured the expression of vWF (Fig. 9B). The result shows that dual modifications of hybridization and heparinization significantly promote the expression level of vWF in comparison to CA and CA/BNC. The vWF is a glycoprotein produced in Weibel-Palade bodies within the cytoplasm of ECs [62], which is a vital predictor of endothelialization [63] and plays a significant role in facilitating anticoagulation [64]. The significant up-regulation of vWF observed in the HUVEC cells grown on $\mathrm{CA} / \mathrm{BNC}-\mathrm{H} 3$ further approves the favorable role of dual modifications in 

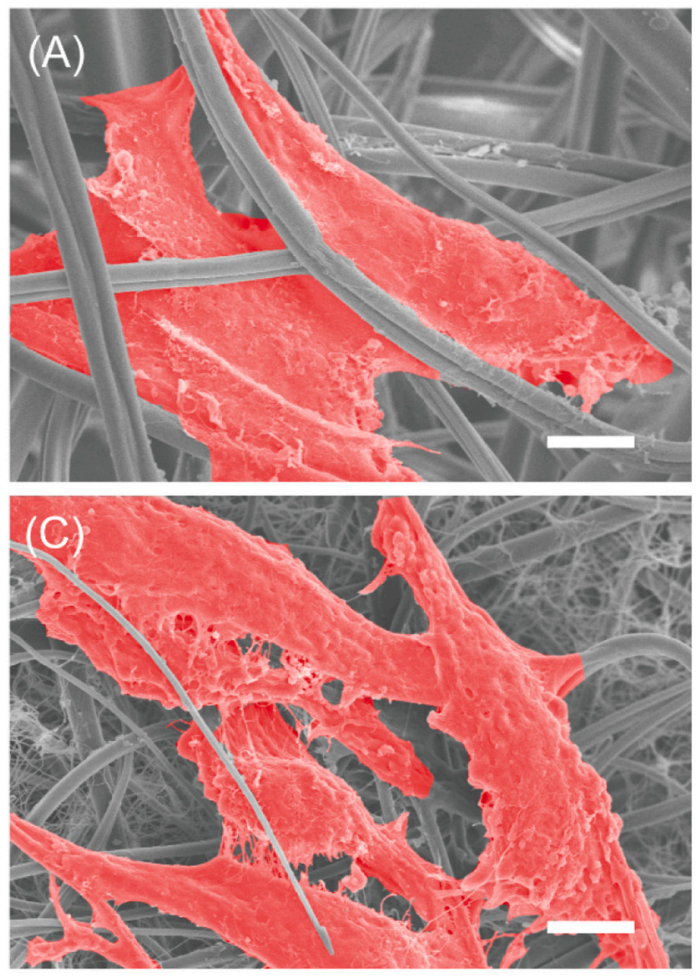

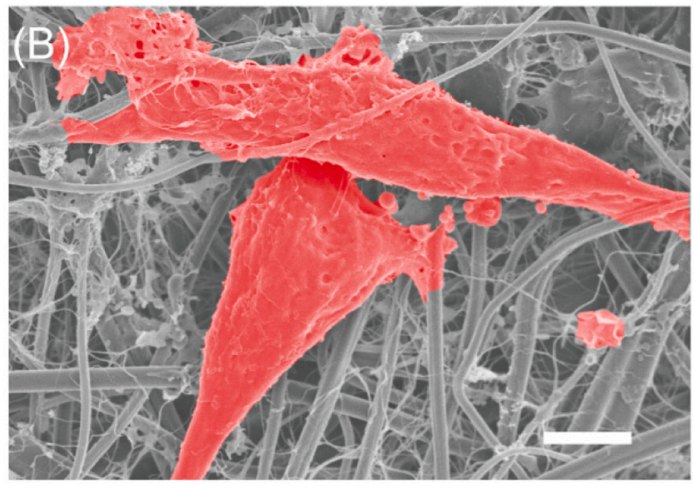

(D)

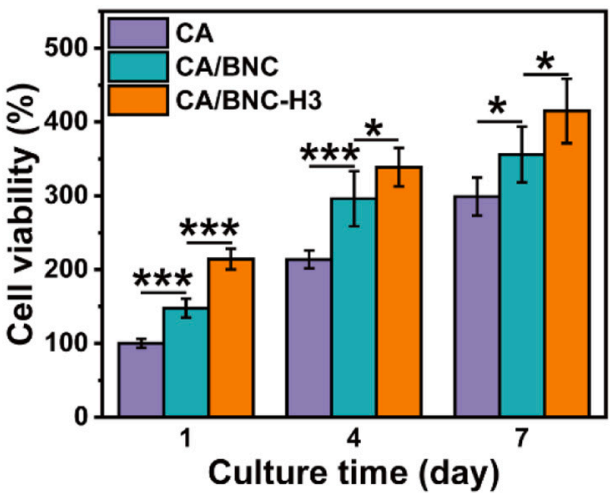

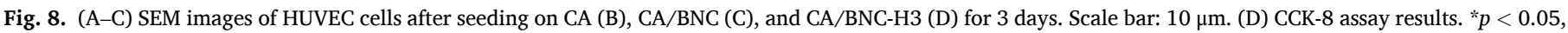
$* * * p<0.001, \mathrm{n}=5$.
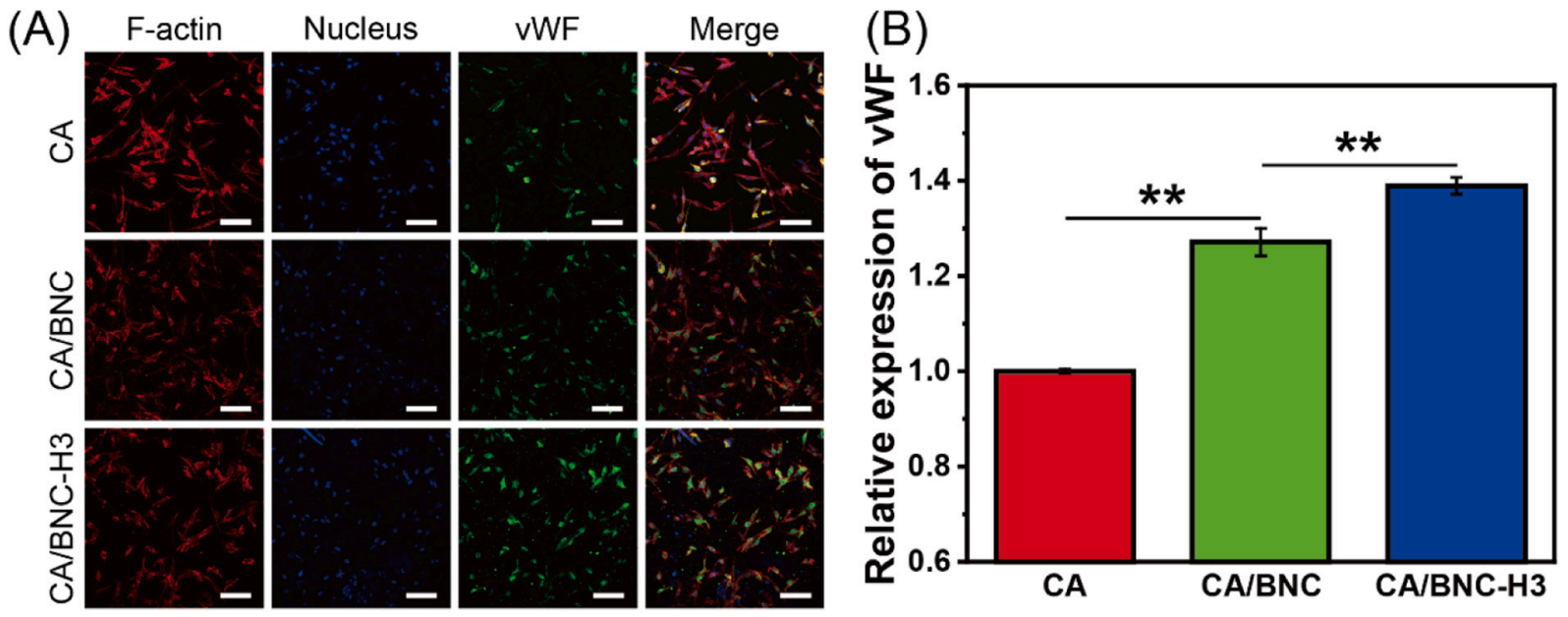

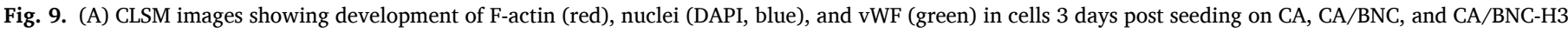

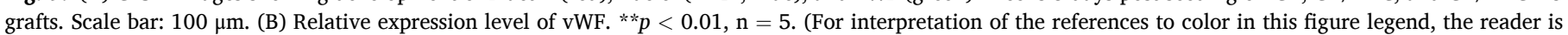
referred to the web version of this article.)

promoting endothelialization and anticoagulation.

Besides endothelialization, anticoagulation function was assessed as well. It is noted that the incorporation of BNC nanofibers to electrospun CA microfibers increases platelet adhesion due to the improved hydrophilicity of CA/BNC graft (Fig. S4) [65,66]. Fortunately, CA/BNC-H3 shows a significant reduction in platelet adhesion when compared to CA and CA/BNC (Fig. 6), suggesting the significant role of heparin in inhibiting platelet adhesion.

All these results indicate that the CA/BNC-H3 can not only promote cell attachment and proliferation due to ECM-like structure, but also enhance antithrombogenic behavior through heparin immobilization. The mechanism is illustrated in Fig. 10.
Besides excellent blood compatibility and endothelialization, matched mechanical performance to withstand the pressure of flowing blood is another critical requirement [67]. Although CA shows a relatively low mechanical strength, CA/BNC and, particularly, CA/BNC-H3 show sufficient mechanical performance. The mechanical testing results (Fig. 4) suggest that immobilizing heparin on fibrous materials can lead to improvements in strength, likely due to the interfacial interactions between heparin and CA/BNC as revealed by FTIR results (Fig. 3). Note that there is no difference in tensile modulus $(p>0.05)$ between CA/ $\mathrm{BNC}$ and CA/BNC-H grafts. The tensile strength of CA/BNC-H3 is larger than the maximum stress of coronary arteries (1.44 $\pm 0.87 \mathrm{MPa})$ measured by Karimi et al. [68], and almost equals to the fracture 


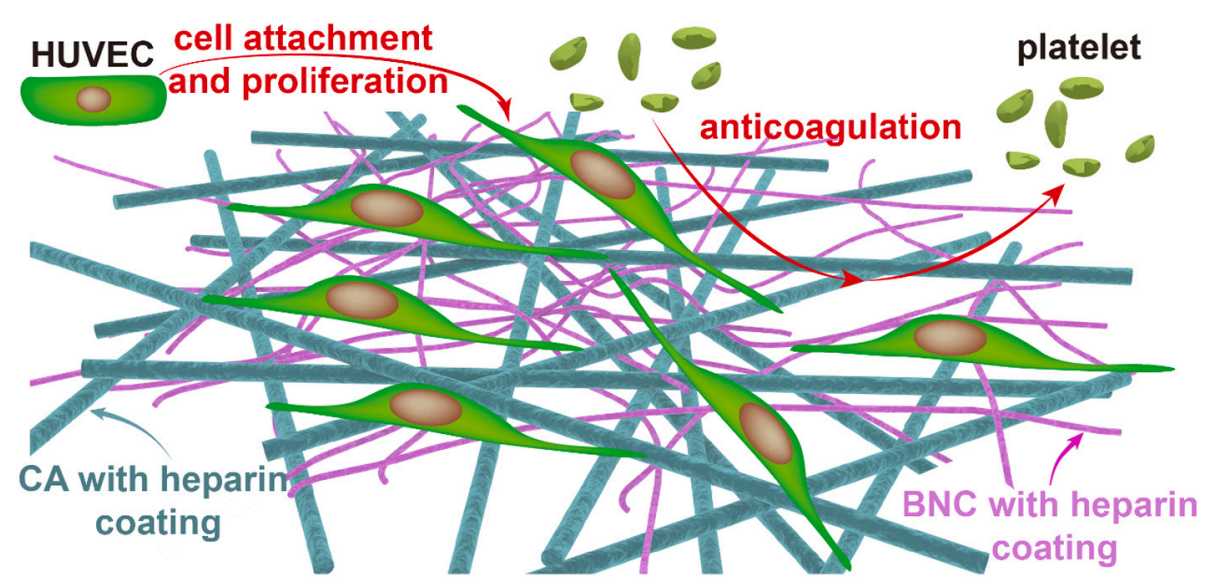

Fig. 10. Schematic illustration showing the combined effects of nano-micro-fibrous structure that favors ECs attachment and proliferation and heparin that inhibits platelets adhesion.

strength of human thoracic aorta (about 1.95 MPa) and the fracture strength of human femoral artery (1-2 MPa) [69]. The Young's modulus is higher than that of coronary artery (0.7-2.1 MPa) [70], and the strain is comparable to that of native thoracic aorta (48\%) [70]. These results indicate that the mechanical properties of CA/BNC-H3 are sufficient for a graft. In conjunction with the favorable blood compatibility and endothelialization, such mechanically matched graft shows promising in future applications in replacing damaged and diseased small-diameter blood vessels although further studies are required to evaluate their endothelialization in vivo and long-term patency.

\section{Conclusions}

BNC nanofibers were incorporated into the electrospun microfibrous CA graft to endow it with biomimetic architecture. The CA and BNC fibers in the CA/BNC graft were immobilized with heparin. The hybridized and heparinized CA/BNC-H graft maintains the porous structure of bare CA and BNC grafts with an average pore size of around 10 $\mu \mathrm{m}$, which facilitates EC adhesion and proliferation and communication between ECs and SMCs, but inhibits fibrous tissue infiltration and prevents blood leakage. The immobilized heparin on BNC and CA fibers exhibits sustained release yet an initial burst release is noted. The mechanical properties of the CA/BNC-H graft are greatly improved compared with bare CA graft and larger than CA/BNC, which are sufficient as a mechanically matched graft. It is found that hybridization with BNC nanofibers cannot improve blood compatibility of CA graft while heparin coating greatly inhibits platelet adhesion and activation. Fluorescence microscopy and CLSM images of HUVEC cells seeded on $\mathrm{CA}, \mathrm{CA} / \mathrm{BNC}$, and CA/BNC-H grafts suggest that either hybridization or heparinization can promote cell attachment and proliferation. The CA/ BNC-H graft with combined advantages of biomimetic architecture and heparin coating exhibits enhanced endothelialization and blood compatibility compared to CA and CA/BNC grafts. The method described here provides a simple and effective way for engineering vascular grafts with excellent blood compatibility and complete endothelialization as well as compatible mechanical properties yet the in vivo investigation on the patency of the novel CA/BNC-H graft is required in future studies.

\section{CRediT authorship contribution statement}

Quanchao Zhang: Validation, Writing - Original draft.

Shan He: Investigation.

Xiangbo Zhu: Visualization.

Honglin Luo: Conceptualization, Funding acquisition, Writing original draft.
Miguel Gama: Formal analysis.

Mengxia Peng: Investigation.

Xiaoyan Deng: Methodology, Project administration.

Yizao Wan: Writing - Review \& editing, Funding acquisition, Supervision.

\section{Declaration of competing interest}

The authors declare that they have no known competing financial interests or personal relationships that could have appeared to influence the work reported in this paper.

\section{Acknowledgements}

This work was supported by the National Natural Science Foundation of China (grant nos. 51973058 and 31870963), the Key Research and Development Program of Jiangxi Province (No. 20192ACB80008), and the Key Project of Natural Science Foundation of Jiangxi Province (20202ACBL204013).

\section{Appendix A. Supplementary data}

Supplementary data to this article can be found online at https://doi. org/10.1016/j.msec.2020.111861.

\section{References}

[1] B. Al Meslmani, G. Mahmoud, B. Strehlow, E. Mohr, T. Leichtweiss, U. Bakowsky, Development of thrombus-resistant and cell compatible crimped polyethylene terephthalate cardiovascular grafts using surface co-immobilized heparin and collagen, Mater. Sci. Eng. C 43 (2014) 538-546.

[2] A.I. Cassady, N.M. Hidzir, L. Grondahl, Enhancing expanded poly (tetrafluoroethylene) (ePTFE) for biomaterials applications, J. Appl. Polym. Sci. 131 (15) (2014) 40533.

[3] X. Ren, Y. Feng, J. Guo, H. Wang, Q. Li, J. Yang, X. Hao, J. Lv, N. Ma, W. Li, Surface modification and endothelialization of biomaterials as potential scaffolds for vascular tissue engineering applications, Chem. Soc. Rev. 44 (15) (2015) 5680-5742.

[4] P. Gupta, K.L. Lorentz, D.G. Haskett, E.M. Cunnane, A.K. Ramaswamy, J. S. Weinbaum, D.A. Vorp, B.B. Mandal, Bioresorbable silk grafts for small diameter vascular tissue engineering applications: in vitro and in vivo functional analysis, Acta Biomater. 105 (2020) 146-158.

[5] M. Kimicata, J.D. Allbritton-King, J. Navarro, M. Santoro, T. Inoue, N. Hibino, J. P. Fisher, Assessment of decellularized pericardial extracellular matrix (ECM) and poly(propylene fumarate) (PPF) biohybrid for small-diameter vascular graft applications, Acta Biomater. 110 (2020) 68-81.

[6] X. Peng, X. Wang, C. Cheng, X. Zhou, Z. Gu, L. Li, J. Liu, X. Yu, Bioinspired, artificial, small-diameter vascular grafts with selective and rapid endothelialization based on an amniotic membrane-derived hydrogel, ACS Biomater. Sci. Eng. 6 (3) (2020) 1603-1613. 
[7] T. Asakura, T. Tanaka, R. Tanaka, Advanced silk fibroin biomaterials and application to small-diameter silk vascular grafts, ACS Biomater. Sci. Eng. 5 (11) (2019) 5561-5577.

[8] Y. Wang, C. He, Y. Feng, Y. Yang, Z. Wei, W. Zhao, C. Zhao, A chitosan modified asymmetric small-diameter vascular graft with anti-thrombotic and anti-bacterial functions for vascular tissue engineering, J. Mater. Chem. B 8 (3) (2020) 568-577.

[9] S. Yan, B. Napiwocki, Y. Xu, J. Zhang, X. Zhang, X. Wang, W.C. Crone, Q. Li, L. S. Turng, Wavy small-diameter vascular graft made of eggshell membrane and thermoplastic polyurethane, Mater. Sci. Eng. C 107 (2020) 110311.

[10] H.Y. Mi, Y. Jiang, X. Jing, E. Enriquez, H. Li, Q. Li, L.S. Turng, Fabrication of triplelayered vascular grafts composed of silk fibers, polyacrylamide hydrogel, and polyurethane nanofibers with biomimetic mechanical properties, Mater. Sci. Eng. C 98 (2019) 241-249.

[11] X. Wang, B. Ding, B. Li, Biomimetic electrospun nanofibrous structures for tissue engineering, Mater. Today 16 (6) (2013) 229-241.

[12] A. Hasan, A. Memic, N. Annabi, M. Hossain, A. Paul, M.R. Dokmeci, F. Dehghani, A. Khademhosseini, Electrospun scaffolds for tissue engineering of vascular grafts, Acta Biomater. 10 (1) (2014) 11-25.

[13] D. Wang, X. Wang, X. Li, L. Jiang, Z. Chang, Q. Li, Biologically responsive, longterm release nanocoating on an electrospun scaffold for vascular endothelialization and anticoagulation, Mater. Sci. Eng. C 107 (2020) 110212.

[14] Q. Hu, C. Wu, H. Zhang, Preparation and optimization of a gelatin-based biomimetic three-layered vascular scaffold, J. Biomater. Appl. 34 (3) (2019) $431-441$.

[15] J. Ding, J. Zhang, J. Li, D. Li, C. Xiao, H. Xiao, H. Yang, X. Zhuang, X. Chen, Electrospun polymer biomaterials, Prog. Polym. Sci. 90 (2019) 1-34.

[16] J.A. Wood, S.J. Liliensiek, P. Russell, P.F. Nealey, C.J. Murphy, Biophysical cueing and vascular endothelial cell behavior, Materials 3 (3) (2010) 1620-1639.

[17] D. Wang, Y. Xu, Q. Li, L.S. Turng, Artificial small-diameter blood vessels: materials, fabrication, surface modification, mechanical properties, and bioactive functionalities, J. Mater. Chem. B 8 (9) (2020) 1801-1822.

[18] A. Joshi, Z. Xu, Y. Ikegami, S. Yamane, M. Tsurashima, H. Ijima, Co-culture of mesenchymal stem cells and human umbilical vein endothelial cells on heparinized polycaprolactone/gelatin co-spun nanofibers for improved endothelium remodeling, Int. J. Biol. Macromol. 151 (2020) 186-192.

[19] Z. Liu, Z. Zheng, K. Chen, Y. Li, X. Wang, G. Li, A heparin-functionalized woven stent graft for endovascular exclusion, Colloid. Surfaces B 180 (2019) 118-126.

[20] M.J.B. Wissink, R. Beernink, J.S. Pieper, A.A. Poot, G.H.M. Engbers, T. Beugeling, W.G. van Aken, J. Feijen, Immobilization of heparin to EDC/NHS-crosslinked collagen, Characterization and in vitro evaluation, Biomaterials 22 (2) (2001) $151-163$.

[21] W. Wang, D. Liu, D. Li, H. Du, J. Zhang, Z. You, M. Li, C. He, Nanofibrous vascular scaffold prepared from miscible polymer blend with heparin/stromal cell-derived factor-1 alpha for enhancing anticoagulation and endothelialization, Colloid. Surfaces B 181 (2019) 963-972.

[22] Y. Wan, T. Cui, Q. Zhang, Z. Yang, F. Yao, H. Luo, Submicrofiber-incorporated 3D bacterial cellulose nanofibrous scaffolds with enhanced cell performance, Macromol. Mater. Eng. 303 (11) (2018) 1800316.

[23] C. Weber, S. Reinhardt, K. Eghbalzadeh, M. Wacker, M. Guschlbauer, A. Maul, A. Sterner-Kock, T. Wahlers, J. Wippermann, M. Scherner, Patency and in vivo compatibility of bacterial nanocellulose grafts as small-diameter vascular substitute, J. Vasc. Surg. 68 (6) (2018) 177S-187S.

[24] L. Bao, J. Tang, F.F. Hong, X. Lu, L. Chen, Physicochemical properties and in vitro biocompatibility of three bacterial nanocellulose conduits for blood vessel applications, Carbohyd. Polym. 239 (2020) 116246.

[25] K. Novotna, P. Havelka, T. Sopuch, K. Kolarova, V. Vosmanska, V. Lisa, V. Svorcik, L. Bacakova, Cellulose-based materials as scaffolds for tissue engineering, Cellulos 20 (5) (2013) 2263-2278.

[26] H. Luo, J. Dong, Y. Zhang, G. Li, R. Guo, G. Zuo, M. Ye, Z. Wang, Z. Yang, Y. Wan, Constructing 3D bacterial cellulose/graphene/polyaniline nanocomposites by novel layer-by-layer in situ culture toward mechanically robust and highly flexible freestanding electrodes for supercapacitors, Chem. Eng. J. 334 (2018) 1148-1158.

[27] H. Si, H. Luo, G. Xiong, Z. Yang, S.R. Raman, R. Guo, Y. Wan, One-step in situ biosynthesis of graphene oxide-bacterial cellulose nanocomposite hydrogels, Macromol. Rapid Commun. 35 (9) (2014) 1706-1711.

[28] H. Luo, P. Xiong, J. Xie, Z. Yang, Y. Huang, J. Hu, Y. Wan, Y. Xu, Uniformly dispersed freestanding carbon nanofiber/graphene electrodes made by a scalable biological method for high-performance flexible supercapacitors, Adv. Funct. Mater. 28(0) (2018) 1803075.

[29] H. Luo, J. Dong, X. Xu, J. Wang, Z. Yang, Y. Wan, Exploring excellent dispersion of graphene nanosheets in three-dimensional bacterial cellulose for ultra-strong nanocomposite hydrogels, Compos. Part A 109 (2018) 290-297.

[30] Y. Wan, S. Yang, J. Wang, D. Gan, M. Gama, Z. Yang, Y. Zhu, F. Yao, H. Luo, Scalable synthesis of robust and stretchable composite wound dressings by dispersing silver nanowires in continuous bacterial cellulose, Compos. Part B 199 (2020) 108259.

[31] H. Luo, D. Gan, M. Gama, J. Tu, F. Yao, Q. Zhang, H. Ao, Z. Yang, J. Li, Y. Wan, Interpenetrated nano-and submicro-fibrous biomimetic scaffolds toward enhanced mechanical and biological performances, Mater. Sci. Eng. C 108 (2020), 110416.

[32] Y. Wan, Z. Lin, Q. Zhang, D. Gan, M. Gama, J. Tu, H. Luo, Incorporating graphene oxide into biomimetic nano-microfibrous cellulose scaffolds for enhanced breast cancer cell behavior, Cellulose 27 (8) (2020) 4471-4485.

[33] D. Zhou, C. Too, G. Wallace, Synthesis and characterisation of polypyrrole/heparin composites, React. Funct. Polym. 39 (1) (1999) 19-26.
[34] P. da Luz Moreira, M.L.F. Wada, W.P. Novello, Importance of uniform heparin coating on biopolymers, Artif. Organs 24 (3) (2000) 209-211.

[35] N. Yin, R. Du, F. Zhao, Y. Han, Z. Zhou, Characterization of antibacterial bacterial cellulose composite membranes modified with chitosan or chitooligosaccharide, Carbohyd. Polym. 229 (2019).

[36] X.M. Wu, C.J. Branford-White, L.M. Zhu, N.P. Chatterton, D.G. Yu, Ester prodrugloaded electrospun cellulose acetate fiber mats as transdermal drug delivery systems, J. Mater. Sci. Mater. Med. 21 (8) (2010) 2403-2411.

[37] A.S.M. Ali, M.R. El-Aassar, F.S. Hashem, N.A. Moussa, Surface modified of cellulose acetate electrospun nanofibers by polyaniline/ $\beta$-cyclodextrin composite for removal of cationic dye from aqueous medium, Fiber. Polym. 20 (10) (2019) 2057-2069.

[38] D. Zhao, Y. Wang, Z. Chen, R. Xu, G. Wu, D. Yu, Preparation and characterization of modified hydroxyapatite particles by heparin, Biomed. Mater. 3 (2) (2008), 025016.

[39] N.F. Vasconcelos, J.P.A. Feitosa, F.M.P. da Gama, J.P.S. Morais, F.K. Andrade, M.d. S.M. de Souza Filho, M.d.F. Rosa, Bacterial cellulose nanocrystals produced under different hydrolysis conditions: properties and morphological features, Carbohyd. Polym. 155 (2017) 425-431.

[40] Y. Maréchal, H. Chanzy, The hydrogen bond network in I $\beta$ cellulose as observed by infrared spectrometry, J. Mol. Struct. 523 (1) (2000) 183-196.

[41] Y. Sun, C. Meng, Y. Zheng, Y. Xie, W. He, Y. Wang, K. Qiao, L. Yue, The effects of two biocompatible plasticizers on the performance of dry bacterial cellulose membrane: a comparative study, Cellulose 25 (10) (2018) 5893-5908.

[42] W. Li, X. Li, W. Li, T. Wang, X. Li, S. Pan, H. Deng, Nanofibrous mats layer-by-layer assembled via electrospun cellulose acetate and electrosprayed chitosan for cell culture, Eur. Polym. J. 48 (11) (2012) 1846-1853.

[43] Y.C. Ho, F.L. Mi, H.W. Sung, P.L. Kuo, Heparin-functionalized chitosan-alginate scaffolds for controlled release of growth factor, Int. J. Pharm. 376 (1-2) (2009) 69-75.

[44] D. Grant, W.F. Long, F.B. Williamson, Infrared spectroscopy of heparin-cation complexes, Biochem. J. 244 (1) (1987) 143-149.

[45] W. Marconi, A. Galloppa, A. Martinelli, A. Piozzi, New polyurethane compositions able to bond high amounts of both albumin and heparin: II: copolymers and polymer blends, Biomaterials 17 (18) (1996) 1795-1802.

[46] X. Li, J. Tang, L. Bao, L. Chen, F.F. Hong, Performance improvements of the BNC tubes from unique double-silicone-tube bioreactors by introducing chitosan and heparin for application as small-diameter artificial blood vessels, Carbohyd. Polym. 178 (2017) 394-405.

[47] D.H. Go, Y.K. Joung, S.Y. Park, Y.D. Park, K.D. Park, Heparin-conjugated starshaped PLA for improved biocompatibility, J. Biomed. Mater. Res. A 86 (3) (2008) $842-848$.

[48] E.S. Sashina, G. Janowska, M. Zaborski, A.V. Vnuchkin, Compatibility of fibroin/ chitosan and fibroin/cellulose blends studied by thermal analysis, J. Therm. Anal. Calorim. 89 (3) (2007) 887-891.

[49] S.M. Li, N. Jia, J.F. Zhu, M.G. Ma, R.C. Sun, Synthesis of cellulose-calcium silicate nanocomposites in ethanol/water mixed solvents and their characterization, Carbohyd. Polym. 80 (1) (2010) 270-275.

[50] A.F. Martins, A.G. Pereira, A.R. Fajardo, A.F. Rubira, E.C. Muniz, Characterization of polyelectrolytes complexes based on $\mathrm{N}, \mathrm{N}, \mathrm{N}$-trimethyl chitosan/heparin prepared at different pH conditions, Carbohyd. Polym. 86 (3) (2011) 1266-1272.

[51] Q. Lu, S. Zhang, K. Hu, Q. Feng, C. Cao, F. Cui, Cytocompatibility and blood compatibility of multifunctional fibroin/collagen/heparin scaffolds, Biomaterials 28 (14) (2007) 2306-2313.

[52] W. Yao, H. Gu, T. Hong, Y. Wang, S. Chen, X. Mo, W. Li, C. Wang, T. Zhu, S. Lu, A bi-layered tubular scaffold for effective anti-coagulant in vascular tissue engineering, Mater. Design 194 (2020) 108943.

[53] D. Hao, Y. Fan, W. Xiao, R. Liu, C. Pivetti, T. Walimbe, F. Guo, X. Zhang, D. L. Farmer, F. Wang, A. Panitch, K.S. Lam, A. Wang, Rapid endothelialization of small diameter vascular grafts by a bioactive integrin-binding ligand specifically targeting endothelial progenitor cells and endothelial cells, Acta Biomater. 108 (2020) 178-193.

[54] S. Oliveira, T. Felizardo, S. Amorim, S.M. Mithieux, R.A. Pires, R.L. Reis, A. Martins, A.S. Weiss, N.M. Neves, Tubular fibrous scaffold functionalized with tropoelastin as a small-diameter vascular graft, Biomacromolecules 21 (9) (2020) 3582-3595.

[55] F.H. Silver, P.B. Snowhill, D.J. Foran, Mechanical behavior of vessel wall: a comparative study of aorta, vena cava, and carotid artery, Ann. Biomed. Eng. 31 (7) (2003) 793-803.

[56] T. Jungst, I. Pennings, M. Schmitz, A.J. Rosenberg, J. Groll, D. Gawlitta, Heterotypic scaffold design orchestrates primary cell organization and phenotypes in cocultured small diameter vascular grafts, Adv. Funct. Mater. 29 (43) (2019), 1905987.

[57] S.H. Ku, C.B. Park, Human endothelial cell growth on mussel-inspired nanofiber scaffold for vascular tissue engineering, Biomaterials 31 (36) (2010) 9431-9437.

[58] A. Goins, A.R. Webb, J.B. Allen, Multi-layer approaches to scaffold-based small diameter vessel engineering: a review, Mater. Sci. Eng. C 97 (2019) 896-912.

[59] M. Ahmed, H. Ghanbari, B.G. Cousins, G. Hamilton, A.M. Seifalian, Small calibre polyhedral oligomeric silsesquioxane nanocomposite cardiovascular grafts: influence of porosity on the structure, haemocompatibility and mechanical properties, Acta Biomater. 7 (11) (2011) 3857-3867.

[60] Z. Yang, O. Tu, J. Wang, N. Huang, The role of heparin binding surfaces in the direction of endothelial and smooth muscle cell fate and re-endothelialization, Biomaterials 33 (28) (2012) 6615-6625. 
[61] Y. Ding, M. Yang, Z. Yang, R. Luo, X. Lu, N. Huang, P. Huang, Y. Leng, Cooperative control of blood compatibility and re-endothelialization by immobilized heparin and substrate topography, Acta Biomater. 15 (2015) 150-163.

[62] D.B. Cines, E.S. Pollak, C.A. Buck, J. Loscalzo, G.A. Zimmerman, R.P. McEver, J. S. Pober, T.M. Wick, B.A. Konkle, B.S. Schwartz, E.S. Barnathan, K.R. McCrae, B. A. Hug, A.M. Schmidt, D.M. Stern, Endothelial cells in physiology and in the pathophysiology of vascular disorders, Blood 91 (10) (1998) 3527-3561.

[63] Y. Xie, M. Takano, D. Murakami, M. Yamamoto, K. Okamatsu, S. Inami, K. Seimiya, T. Ohba, Y. Seino, K. Mizuno, Comparison of neointimal coverage by optical coherence tomography of a sirolimus-eluting stent versus a bare-metal stent three months after implantation, Am. J. Cardiol. 102 (1) (2008) 27-31.

[64] F. Du, W. Zhao, M. Zhang, H. Mao, D. Kong, J. Yang, The synergistic effect of aligned nanofibers and hyaluronic acid modification on endothelial cell behavior for vascular tissue engineering, J. Nanosci. Nanotechnol. 11 (8) (2011) 6718-6725.

[65] S. Movafaghi, V. Leszczak, W. Wang, J.A. Sorkin, L.P. Dasi, K.C. Popat, A.K. Kota Hemocompatibility of superhemophobic titania surfaces, Adv. Healthcare Mater. 6 (4) (2017) 1600717.
[66] L. Zhang, B. Casey, D.K. Galanakis, C. Marmorat, S. Skoog, K. Vorvolakos, M. Simon, M.H. Rafailovich, The influence of surface chemistry on adsorbed fibrinogen conformation, orientation, fiber formation and platelet adhesion, Acta Biomater. 54 (2017) 164-174.

[67] K. Liu, N. Wang, W. Wang, L. Shi, H. Li, F. Guo, L. Zhang, L. Kong, S. Wang, Y. Zhao, A bio-inspired high strength three-layer nanofiber vascular graft with structure guided cell growth, J. Mater. Chem. B 5 (20) (2017) 3758-3764.

[68] A. Karimi, M. Navidbakhsh, A. Shojaei, S. Faghihi, Measurement of the uniaxial mechanical properties of healthy and atherosclerotic human coronary arteries, Mater. Sci. Eng. C 33 (5) (2013) 2550-2554.

[69] W. He, T. Yong, W.E. Teo, Z.W. Ma, S. Ramakrishna, Fabrication and endothelialization of collagen-blended biodegradable polymer nanofibers: potential vascular graft for blood vessel tissue engineering, Tissue Eng. 11 (9-10) (2005) 1574-1588.

[70] S.K. Norouzi, A. Shamloo, Bilayered heparinized vascular graft fabricated by combining electrospinning and freeze drying methods, Mater. Sci. Eng. C 94 (2019) 1067-1076. 